\title{
Hommel, Mandy
}

\section{Prozessorientiertes Lernhandeln mit ERP-Software}

Wittmann, Eveline [Hrsg.]; Frommberger, Dietmar [Hrsg.]; Ziegler, Birgit [Hrsg.]: Jahrbuch der berufs- und wirtschaftspädagogischen Forschung 2018. Opladen ; Berlin ; Toronto : Verlag Barbara Budrich 2018, S. 53-66. (Schriftenreihe der Sektion Berufs- und Wirtschaftspädagogik der Deutschen Gesellschaft für Erziehungswissenschaft (DGfE))

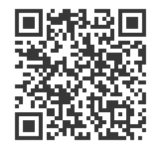

Quellenangabe/ Reference:

Hommel, Mandy: Prozessorientiertes Lernhandeln mit ERP-Software - In: Wittmann, Eveline [Hrsg.]; Frommberger, Dietmar [Hrsg.]; Ziegler, Birgit [Hrsg.]: Jahrbuch der berufs- und wirtschaftspädagogischen Forschung 2018. Opladen ; Berlin ; Toronto : Verlag Barbara Budrich 2018, S. 53-66 - URN: urn:nbn:de:0111-pedocs-183556 - DOI: 10.25656/01:18355

https://nbn-resolving.org/urn:nbn:de:0111-pedocs-183556

https://doi.org/10.25656/01:18355

in Kooperation mit / in cooperation with:

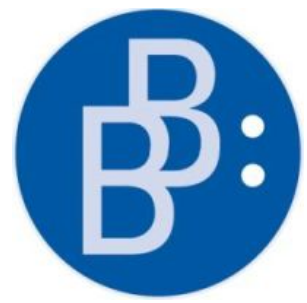

https://www.budrich.de

\section{Nutzungsbedingungen}

Dieses Dokument steht unter folgender Creative Commons-Lizenz: http://creativecommons.org/licenses/by-sa/4.0/deed.de - Sie dürfen das Werk bzw. den Inhalt vervielfältigen, verbreiten und öffentlich zugänglich machen sowie Abwandlungen und Bearbeitungen des Werkes bzw. Inhaltes anfertigen, solange sie den Namen des Autors/Rechteinhabers in der von inm festgelegten Weise nennen und die daraufhin neu entstandenen Werke bzw. Inhalte nur unter Verwendung von Lizenzbedingungen weitergeben, die mit denen dieses Lizenzvertrags identisch, vergleichbar oder kompatibel sind.

Mit der Verwendung dieses Dokuments erkennen Sie die Nutzungsbedingungen an.

\section{Terms of use}

This document is published under following Creative Commons-License: http://creativecommons.org/licenses/by-sa/4.0/deed.en - You may copy, distribute and transmit, adapt or exhibit the work or its contents in public and alter, transform, or change this work as long as you attribute the work in the manner specified by the author or licensor. New resulting works or contents must be distributed pursuant to this license or an identical or comparable license.

By using this particular document, you accept the above-stated conditions of use.

\section{Kontakt / Contact:}

\section{peDOCS}

DIPF | Leibniz-Institut für Bildungsforschung und Bildungsinformation Informationszentrum (IZ) Bildung

E-Mail: pedocs@dipf.de

Internet: www.pedocs.de

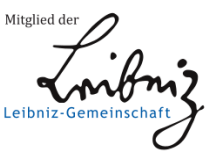




\section{Jahrbuch der berufs- und wirtschaftspädagogischen Forschung 2018}

Eveline Wittmann, Dietmar Frommberger, Birgit Ziegler (Hrsg.)

\section{DEE Deutsche Gesellschaft}


Eveline Wittmann

Dietmar Frommberger

Birgit Ziegler (Hrsg.)

\section{Jahrbuch der berufs- und wirtschaftspädagogischen Forschung 2018}

Verlag Barbara Budrich

Opladen • Berlin • Toronto 2018 
Bibliografische Information der Deutschen Nationalbibliothek

Die Deutsche Nationalbibliothek verzeichnet diese Publikation in der Deutschen

Nationalbibliografie; detaillierte bibliografische Daten sind im Internet über

http://dnb.d-nb.de abrufbar.

(C) 2018 Dieses Werk ist beim Verlag Barbara Budrich erschienen und steht unter der Creative Commons Lizenz Attribution-ShareAlike 4.0 International (CC BY-SA 4.0): https://creativecommons.org/licenses/by-sa/4.0/

Diese Lizenz erlaubt die Verbreitung, Speicherung, Vervielfältigung und Bearbeitung bei Verwendung der gleichen CC-BY-SA 4.0-Lizenz und unter Angabe der UrheberInnen, Rechte, Änderungen und verwendeten Lizenz.

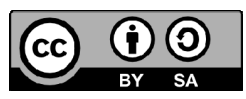

Dieses Buch steht im Open-Access-Bereich der Verlagsseite zum kostenlosen Download bereit (https://doi.org/10.3224/84742223).

Eine kostenpflichtige Druckversion (Print on Demand) kann über den Verlag bezogen werden. Die Seitenzahlen in der Druck- und Onlineversion sind identisch.

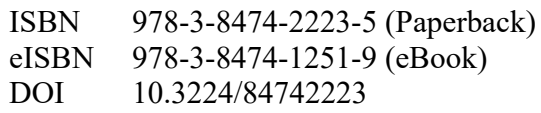

Umschlaggestaltung: Bettina Lehfeldt, Kleinmachnow - www.lehfeldtgraphic.de Technisches Lektorat: Linda Kutzki, Berlin - www.textsalz.de Druck: paper \& tinta, Warschau Printed in Europe 


\section{Inhaltsverzeichnis}

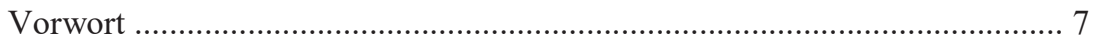

\section{Teil I: Sichtweisen des Lehr- und Ausbildungspersonals}

Tobias Kärner, Julia Warwas und Karin Heinrichs

Sichtweisen angehender Berufsschullehrkräfte und Ausbildender auf

Lehren und Lernen: Welche Rolle spielen erlebter elterlicher

Erziehungsstil und erlebter Unterricht

Ariane Neu

Gestaltungsoptionen zur Stärkung beruflicher Aus- und Fortbildung aus

Sicht betrieblicher Akteure

\section{Teil II: Berufliche Didaktik und Kompetenzentwicklung}

Christina Keimes, Volker Rexing und Jens Drescher

Schülervorstellungen als Ausgangspunkt inklusiven Fachunterrichts in bautechnischen Ausbildungsberufen

Mandy Hommel

Prozessorientiertes Lernhandeln mit ERP-Software

Rico Hermkes, Hanna Mach und Gerhard Minnameier

Scaffolding von Problemlöseprozessen im Buchführungsunterricht

Anh Dinh, Marina Haves und Thomas Retzmann

Krisen kompetent bewältigen? Entwicklung eines Kompetenzmodells für die Entrepreneurship Education

\section{Teil III: Berufswahl und Berufslaufbahn}

Sebastian Kirchknopf und Kristina Kögler

Die Bedeutung der Laufbahnadaptabilität für den berufs- und wirtschaftspädagogischen Diskurs. Konstruktverständnis und Forschungsdesiderate 95

Svenja Ohlemann und Angela Ittel

Normwerte der Berufswahlkompetenz: Eine diagnostische Chance zur individuellen Förderung? 


\section{Ulrich Weiß}

„Didaktische Hilflosigkeit“ der Jungarbeiterbeschulung revisited -

Anerkennungstheoretische Analysen des Handelns im Übergangsraum ... 125

Elisabeth Maué, Stephan Schumann und Claudia Diehl

Bildungshintergrund und Bildungspläne geflüchteter Jugendlicher im

System der beruflichen Bildung

\section{Michael Jüttler und Stephan Schumann}

Führen gute schulische Wirtschaftskenntnisse zu einem

Wirtschaftsstudium? Eine Längsschnittstudie zum Einfluss

ökonomischer Kompetenzen von Lernenden auf die Aspiration und

Wahl eines wirtschaftswissenschaftlichen Studiums

\section{Teil IV: Institutionalisierung beruflicher Bildung}

\section{Frank Ragutt}

Das Verhältnis von Berufsbildungswissen und Berufsbildungspolitik am Beispiel der Reformdiskussion zur Wirtschaftsoberschule resp.

Wirtschaftsgymnasium im Spiegel des KMK-Schulausschusses, 1949 und 1969

Rudolf Schröder, Rebecca Lembke und Tina Fletemeyer

Konzeptionelle Gestaltung der Berufs- und Studienorientierung in gymnasialen Schulformen. Eine qualitative Studie zur unterrichtlichen und außerunterrichtlichen Realisierung

Alexandra Dehmel

Existenz, Design und Potenziale arbeitsplatzbasierten Lernens zur

(Wieder-)Eingliederung gering Qualifizierter in den Arbeitsmarkt:

Politik und Praxis in verschiedenen europäischen Ländern

Herausgeberschaft 


\title{
Prozessorientiertes Lernhandeln mit ERP-Software
}

\author{
Mandy Hommel
}

\section{Einleitung}

Reale Produktionsprozesse werden zunehmend digital gesteuert und evolvieren zur smarten Produktion. Reale und virtuelle Objekte sind dabei in CyberPhysical Systems (CPS) über Informationsnetze verbunden und interagieren digital (Baheti \& Gill 2011). Um diese Vision in Unternehmen umzusetzen und effizient zu gestalten, ist eine prozessorientierte Ausrichtung der Unternehmen erforderlich (Gaitanides \& Ackermann 2004). Unternehmen müssen dazu über die erforderliche informationstechnische Infrastruktur verfügen. Neben entsprechender Hardware ist die Software erforderlich, mit der Geschäftsprozesse in geeigneter Weise gesteuert und abgebildet werden können. Die Ausrichtung auf funktionsübergreifende und digital gesteuerte Geschäftsprozesse hat Konsequenzen für die berufliche Arbeit und Bildung. Wo vormals betriebliche Funktionen die Arbeitsorganisation prägten, findet man nun die Orientierung an Geschäftsprozessen (Rebmann \& Tenfelde 2008). In der beruflichen Bildung dienen Arbeits- und Geschäftsprozesse als curricularer Referenzrahmen für Lehr-Lern-Prozesse (Rebmann \& Schlömer 2009).

Problem- und handlungsorientierte Lernumgebungen simulieren situiert und realitätsbezogen die Steuerung von Geschäftsprozessen mithilfe integrierter Unternehmenssoftware (vgl. Sembill \& Frötschl 2018). Solche Lernumgebungen ermöglichen es den Lernenden, ein geschäftsprozessorientiertes Unternehmensverständnis zu entwickeln. Anstatt so handlungsorientiertes und arbeitsanaloges Lernen zu ermöglichen (Gudjons 2014; Tramm 2009), sind in der Praxis jedoch eher „halbherzige Schulungen“ (Hilgenberg 2014) zu Enterprise Resource Planning-Software (ERP-Software) in Form von einfachen Klick- und Orientierungskursen zu beobachten. Allerdings ist die Einführung einer ERP-Software in Unternehmen häufig mit Problemen verbunden (Jansen, Müller, Prümper \& Stein 2005), die aus erforderlichen organisatorischen Anpassungen, veränderten Prozessabläufen, Abstimmungsproblemen bei der Softwareeinführung etc. resultieren können (Hilgenberg, 2014). Ein Erfolgsaspekt ist die Qualifizierung zukünftiger Anwender/-innen. Es ist davon auszugehen, dass ein erfolgreiches Handeln in Geschäftsprozessen die umfassende Kenntnis dieser voraussetzt. Der erfolgreiche Umgang mit ERP-Software erfordert damit - neben der Kenntnis der Softwarefunktionalität - insbesondere ein Verständnis für die zu steuernden Geschäftsprozesse, die bereits im Lernprozess erfahrbar sein sollten. Für Unternehmen stellt sich daher nicht 
nur die Frage, welche Software geeignet ist, sondern auch die didaktische Frage, wie potenzielle Anwender/-innen den Umgang mit ERP-Software erlernen sollten (Sembill \& Frötschl 2018). In diesem Kontext ist auch über die soziale Organisation des Lernhandelns zu entscheiden. Dazu ist z. B. zu hinterfragen, inwiefern die soziale Interaktion mit einem Lernpartner motivieren und das Lernen unterstützen kann (Konrad 2014).

Ein komplexes Forschungsprojekt widmet sich daher der Frage, mit welchem didaktischen Orientierungsrahmen Lernhandeln mit ERP-Software besser gefördert werden kann: mit problemorientierten Lernumgebungen, orientiert an ganzheitlichen Geschäftsprozessen, oder durch traditionelle Klickschulungen, die schrittweise mit den Softwarefunktionen vertraut machen. Dieser Beitrag fokussiert das Erinnern und Anwenden des Gelernten, die soziale Organisation des Lernprozesses und das Verständnis für Geschäftsprozesse.

\section{Prozessorientierung als didaktischer Rahmen}

Die Prozessorientierung, die seit den 1990ern Einzug in die Betriebswirtschaftslehre und in die Aufbauorganisation von Unternehmen gehalten hat, folgt dem Paradigmenwechsel vom Denken in (Unternehmens-)Funktionen hin zu funktionsübergreifenden Geschäftsprozessen (u. a. Pongratz 2012). Betriebliche Prozesse (Ablauforganisation) bestimmen damit maßgeblich die Aufbauorganisation (Gaitanides \& Ackermann 2004). Kernprozesse repräsentieren die „Kernleistung“ (ebd., 16) des Unternehmens und Supportprozesse interne Leistungsprozesse.

Begrifflich ist weiter zwischen Arbeits- und Geschäftsprozessen zu unterscheiden. Während Arbeitsprozessen eine gewerbliche Prozessperspektive im Sinne der Transformation von Material zugrunde liegt, basiert die kaufmännischen Perspektive auf der Steuerung von Informationsströmen in Geschäftsprozessen, die mit den Real- und Nominalgüterströmen und der Wertschöpfung verbunden sind (Tramm 2009). Die Vorgangsketten solcher Prozesse können mithilfe von ERP-Software, für Supportprozesse im Bereich des Personalmanagements bspw. SAP ERP HCM, bearbeitet werden. Um potentielle Anwender/-innen im Umgang mit ERP-Software zu qualifizieren, liegt es nahe, Geschäftsprozesse als didaktischen Rahmen zu nutzen und Lernumgebungen arbeitsanalog zu gestalten. Dazu sind zunächst die relevanten Prozesse zu analysieren, zu strukturieren und Problemstellungen für das Lernhandeln zu konstruieren (Hommel 2017). Lernen kann so ganzheitlich an Arbeits- und Geschäftsprozessen, realer Komplexität und vollständigen Handlungen ausgerichtet werden (Dehnbostel 2007). Ein weiterer Aspekt arbeitsanaloger Gestaltung greift die Kooperation von Mitarbeiter/-innen in Geschäftsprozessen eines Unternehmens auf: die Kooperation mit einem/r Lernpartner/-in. Dadurch 
werden Austausch und Reflexion im Lernprozess ermöglicht (Borsch 2015; Hasselhorn \& Gold 2013). Obwohl in vielen Studien kooperativ Lernende Überlegenheit gegenüber einzeln Lernenden zeigten (u. a. Lou, Abrami \& d'Apollonia 2001; Hattie 2012), sind diese Ergebnisse nicht generalisierbar (Konrad 2014). Zudem wird die Wirksamkeit kooperativen Lernens durch verschiedene Aspekte beeinflusst (Renkl \& Mandl 1995): Lernende müssen über die Fertigkeiten und die Bereitschaft zur Kooperation verfügen sowie zur Kooperation aufgefordert werden (spontane Interaktion ist eher selten; King 2007). Es bedarf zudem anspruchsvoller Lernaufgaben, die Kooperation erfordern (Dubs 2009, 197), und dennoch eine identifizierbare Einzelleistung ermöglichen. Durch eine arbeitsanaloge Ausgestaltung können Lernende in realitätsnahen Strukturen und Prozessen handelnd lernen (Achtenhagen et al. 1992). Eine derart konzipierte, handlungs- und problemorientierte Lernumgebung (u. a. Klauser 1998), die das Handeln in Geschäftsprozessen und die soziale Interaktion mit einem Lernpartner berücksichtigt, macht ERP-Software in einer Simulationsumgebung realitätsnah erfahrbar. Sie berücksichtigt mit Situiertheit und Authentizität, multiplen Kontexten und Perspektiven, Kooperation und instruktionaler Unterstützung die Leitlinien der Gestaltung problemorientierter Lernumgebungen (Reinmann \& Mandl 2006). Aus gemäßigt konstruktivistischer Sicht sollte eine solche Lernumgebung besonders geeignet sein, den Aufbau und die Anwendung neuen Wissens und neuer Fertigkeiten zu unterstützen.

Im Gegensatz zu handlungs- und problemorientierten Lernumgebungen für ERP-Software (nachfolgend als prozessorientiert bezeichnet) sind die in der Praxis häufig anzutreffenden Anwenderschulungen als Klick- und Orientierungsschulungen (nachfolgend als funktionsorientiert bezeichnet) eher darauf ausgerichtet, den potentiellen Nutzer kleinschrittig und i. d. R. ohne Interaktion mit einem Lernpartner mit der Software vertraut zu machen. Lernprozesse sind somit weniger an beruflichen Handlungen (u. a. Tramm 2009), sondern vielmehr an einzelnen Softwarefunktionen und deren Organisationskriterien orientiert (z. B. dem Aufbau des Menübaumes). ERP-Software ist zwar darauf ausgerichtet, die Prozessorientierung zu unterstützen, jedoch müssen Lernprozesse nicht zwangsläufig prozessorientiert konstruiert sein. In diesem Fall besteht jedoch die Gefahr, dass Lernende eine fragmentierte Sicht auf Arbeitsund Geschäftsprozesse entwickeln (Tramm 2009), die einer für erfolgreiches Geschäftsprozessmanagement erforderlichen ganzheitlichen Perspektive auf Geschäftsprozesse abträglich ist. 


\section{Methode}

Mit Blick auf das Vorgenannte sollen folgende Forschungsfragen in diesem Beitrag beantwortet und folgende Hypothesen geprüft werden:

1. Ist prozessorientiertes Lernen funktionsorientiertem Lernen in Bezug auf das Erinnern und Anwenden des Gelernten überlegen? Hypothese $H_{1.1}$ : Prozessorientiert Lernende erreichen einen höheren Lernerfolg als funktionsorientiert Lernende.

2. Erreichen Lernende mit einem Lernpartner (dyadisch) einen höheren Lernerfolg als einzeln Lernende in Bezug auf das Erinnern und Anwenden? Hypothese $H_{2.1}$ : Dyadisch Lernende erreichen einen höheren Lernerfolg als einzeln Lernende.

3. Erreichen einzeln Lernende in der Prozessorientierung einen höheren Lernerfolg als in der Funktionsorientierung? Hypothese $H_{3.1}$ : Einzeln Lernende erreichen einen höheren Lernerfolg in der prozessorientierten als in der funktionsorientierten Bedingung.

4. Erreichen dyadisch Lernende in der Prozessorientierung einen höheren Lernerfolg als in der Funktionsorientierung? Hypothese $H_{4.1}$ : Dyadisch Lernende erreichen einen höheren Lernerfolg in der prozessorientierten als in der funktionsorientierten Bedingung.

Das Vorgehen zur Beantwortung der Forschungsfragen folgt einem 2x2-faktoriellen Design (Abb. 1) mit den Faktoren Lernbedingung (Stufen: funktionsund prozessorientiert) und Sozialform (einzeln und dyadisch Lernende). Dazu wurden zwei Erhebungen durchgeführt. Die erste Studie fand im Dezember 2014 statt. Eine zweite Studie wurde im Dezember 2016 durchgeführt.

Abb. 1: 2x2-faktorielles Design

\begin{tabular}{|c|c|c|c|}
\hline & \multicolumn{2}{|c|}{ Sozialform } \\
\hline & & Dyadisch & Einzeln \\
\hline \multirow{2}{*}{ Lernbedingung } & $\begin{array}{l}\text { Prozessorientiert } \\
\text { (ProzO) }\end{array}$ & PrD & $\operatorname{PrE}$ \\
\hline & $\begin{array}{l}\text { Funktionsorientiert } \\
\text { (FunkO) }\end{array}$ & FoD & FoE \\
\hline
\end{tabular}

Quelle: eigene Darstellung 


\subsection{Stichprobe}

Die Stichprobe der ersten Studie umfasste 100 Studierende $(65 \mathrm{w}, 35 \mathrm{~m}$, Alter $M=23$ ), die zweite Studie 69 Studierende ( $48 \mathrm{w}, 21 \mathrm{~m}$, Alter $M=22$ ) grundständiger wirtschaftswissenschaftlicher Studiengänge der TU Dresden (Tab. 1). Die Aufteilung der Probanden auf die Experimentalgruppen erfolgte zufällig (Rost 2013).

Tab. 1: Übersicht über die Stichprobe in Studie 1 und Studie 2

\begin{tabular}{|c|c|c|c|c|c|c|c|c|c|c|c|c|}
\hline & \multicolumn{7}{|c|}{ Studie 1} & \multicolumn{5}{|c|}{ Studie 2} \\
\hline & & & FoE & FoD & $\operatorname{PrE}$ & $\operatorname{PrD}$ & $\sum$ & FoE & FoD & $\operatorname{PrE}$ & $\operatorname{PrD}$ & $\sum$ \\
\hline Alter & & $M$ & 23 & 23 & 23 & 23 & 23 & 22 & 22 & 23 & 23 & 22 \\
\hline \multirow{2}{*}{ Geschlecht } & & $h$ & 21 & 21 & 9 & 14 & 65 & 15 & 20 & 5 & 8 & 48 \\
\hline & & $h$ & 8 & 10 & 12 & 5 & 35 & 4 & 7 & 3 & 7 & 21 \\
\hline$t$ & & $n$ & 29 & 31 & 21 & 19 & 100 & 19 & 27 & 8 & 15 & 69 \\
\hline
\end{tabular}

Quelle: eigene Darstellung

\subsection{Konzeptionen der Lehrveranstaltungen}

Zwei verschiedene Konzeptionen der Lernumgebung zu SAP ERP HCM kamen zum Einsatz: eine funktionsorientierte und eine prozessorientierte Konzeption (Hommel 2017). Die Lernzeit umfasste jeweils 270 Minuten. Für beide Konzeptionen wurde die IDES AG ${ }^{1}$ als SAP-Modellunternehmen genutzt. Das Lernen im Modell wurde durch das Lernen am Modell in einer begleitenden Vorlesung zu den Grundlagen des Personalmanagements ergänzt (Achtenhagen et al. 1992).

Die funktionsorientierte Konzeption machte die Lernenden anhand der Personal- und Softwarefunktionen kleinschrittig mit der Software vertraut. Der Ausgestaltung liegt eine Anwenderschulung ${ }^{2}$ zugrunde, in der die Lernenden mithilfe stark strukturierter und kleinteiliger Arbeitsaufgaben, die die Vorgehensweise in der Software (z. B. das Kopieren einer Planstelle) abbilden, lernen.

In der prozessorientierten Konzeption lösten die Lernenden in der Rolle eines/r fiktiven Mitarbeiter(s)/in im Personalmanagement komplexe, arbeits-

1 IDES steht für International Demonstration and Education System (SAP, o. D.).

2 Die Anwenderschulung in der funktionsorientierten Konzeption wird seit vielen Jahren in der Hochschullehre der TU Dresden genutzt. Bei der prozessorientierten Konzeption handelt es sich um eine handlungs- und problemorientierte Neukonzeption. 
analoge Problemstellungen der Personalbeschaffung und der Personalbetreuung, basierend auf realitätsnahen Recruiting-Prozessabläufen in Unternehmen (Hommel 2017). Ein Manual zu SAP ERP HCM stand zur Verfügung.

\subsection{Erhebungsinstrumente und Untersuchungsablauf}

Vor Beginn der Lehrveranstaltungen wurden Vorwissen und vorhandene Fähigkeiten im Umgang mit SAP ERP HCM mithilfe eines Pretests erfasst. Eine Woche nach den Lehrveranstaltungen bearbeiteten die Teilnehmenden einen Posttest zum Umgang mit SAP ERP HCM. Pre- und Posttest wurden als Paralleltests konzipiert. Die Fähigkeit zum Umgang mit SAP ERP HCM wurde durch Items verschiedener Dimensionen kognitiver Prozesse (Anderson \& Krathwohl 2001) operationalisiert. Vier Items erforderten das Erinnern von Fakten und Prozeduren (nachfolgend Erinnern ${ }^{3}$ ), vier weitere Items das Anwenden und Analysieren (nachfolgend Anwenden ${ }^{4}$ ). Mit drei zusätzlichen Items im Posttest bewerteten die Probanden die Software, einschließlich wahrgenommener Vor- und Nachteile, schätzten ihre Handlungsfähkeit im Umgang mit SAP ERP HCM ein und reflektierten ihren Lernprozess. Nach den Lehrveranstaltungen wurden retrospektive Interviews mit 16 zufällig ausgewählten Probanden geführt, um Einblick in deren Lernprozesse zu erhalten. Zur Kontrolle weiterer Einfluss- und Erklärungsvariablen kamen ergänzende Instrumente zum Einsatz. Der Fokus dieses Beitrags liegt auf dem Lernerfolg im Umgang mit SAP ERP HCM in den Dimensionen Erinnern und Anwenden des Gelernten.

\subsection{Datenauswertung}

Für die inhaltsanalytische Auswertung der Pre- und Posttests ${ }^{5}$ wurde ein Kodierleitfaden entwickelt. Die Interkoderreliabilität wurde anhand von 160 Items (20 Posttests) geprüft. Die Korrelation der von zwei Kodierern erfassten Werte ist insbesondere vor dem Hintergrund der komplexen Kodieranweisung sehr gut $(r=.979 ; n=160)$. Die Paralleltestreliabilität wurde anhand von vier

3 Zum Beispiel erforderte das erste Testitem das Erinnern der Bedeutung eines Symbols aus SAP ERP HCM. Item 2 fragte nach den Möglichkeiten der Suche in SAP ERP HCM, die verschiedenen Suchfunktionen waren zu erinnern.

4 Anwenden meint das konkrete Handeln; für Item 5 war bspw. der komplexe Geschäftsprozess Recruiting mit verschiedenen Geschäftsvorfällen (Pflegen der Planstelle, Anlegen der Ausschreibung, Erfassen der Bewerberdaten, Einladung der Bewerber, Entscheidung und Vorbereitung der Einstellung, Einpflegen der Mitarbeiterdaten) in SAP abzubilden.

5 Aus der Analyse ausgeschlossen werden mussten einige Datensätze, in denen die Probanden die Anweisung zur Lösung der Aufgabe nicht befolgt und die Eingabepfade in SAP nur lückenhaft angegeben hatten, was zu nicht vergleichbaren Resultaten geführt hätte. Die hier angegebenen Stichprobenwerte erfassen nur die Probanden mit gültigen Testwerten. 
Tests (2 Probanden) geprüft (Korrelation nach Pearson $r=.925$ ). Der Wert ist zufriedenstellend, auch wenn der Umfang der Daten, der dafür herangezogen werden konnte, einschränkend zu berücksichtigen ist. Die retrospektiven Interviews wurden einer zusammenfassenden und strukturierenden Inhaltsanalyse unterzogen (Mayring 2015).

Als Voraussetzung für die weitere Datenanalyse wurden die Verteilungen der Daten der einzelnen Gruppen und Subgruppen geprüft. Im Fall anzunehmender Normalverteilung wurden parametrische, ansonsten nichtparametrische Verfahren genutzt $(\alpha=.05)$.

\section{Ergebnisse}

Für die Testitems, die Erinnern und Anwenden erfordern, waren insgesamt 35 Punkte erreichbar (davon Erinnern 6, Anwenden 29). Über beide Erhebungen hinweg zeigten die Probanden im Pretest insgesamt ein sehr geringes Vorwissen $(M=0.41 ; S D=0.76 ;$ Min $=0 ; \operatorname{Max}=4.00)$. Im Posttest lag das arithmetische Mittel der erreichten Punkte bei 11.7 (SD=5.19; Min =1.00; $M a x=27.00)$. Für den Lernerfolg insgesamt ist damit ein großer Effekt zu konstatieren (Mann-Whitney-U-Test, Annahme der $H_{l}$ bei $Z=-16.166, p=.000$, $\left.d_{\text {Cohens }}=3.045\right)$.

Zur ersten Forschungsfrage: (1) Ist prozessorientiertes Lernen (ProzO) funktionsorientiertem Lernen (FunkO) in Bezug auf das Erinnern und Anwenden des Gelernten überlegen? Für die Gruppen der prozess- und funktionsorientierten Bedingung (Studie 1, Studie 2, über beide Studien aggregiert), ist jeweils folgende Hypothese für den Lernerfolg (Erinnern, Anwenden und insgesamt) zu prüfen: $H_{1.1}$ Prozessorientiert Lernende erreichen einen höheren Lernerfolg als funktionsorientiert Lernende.

In Bezug auf den Lernerfolg insgesamt (Tab. 2) erreichen die Lernenden in ProzO sowohl in Studie 1, als auch in Studie 2 bessere Ergebnisse als die Lernenden in FunkO in Studie 1 und in Studie 2. Für die Dimension Erinnern zeigen sich signifikante Unterschiede mit mittlerem Effekt zugunsten der Lernenden in ProzO in Studie $1\left(H_{l}\right.$ des Mann-Whitney-U Tests ist bei $Z=-2.878$ anzunehmen, $\left.d_{\text {Cohens }}=.614, p=.004\right)$, sowie mit großem Effekt in Studie $2\left(H_{1}\right.$ des Mann-Whitney-U-Tests ist bei $Z=-4.175$ anzunehmen, $d_{\text {Cohens }}=1.282$, $p=.000)$. Für die Lernenden in ProzO liegen die über beide Studien aggregierten Werte des Lernerfolgs insgesamt $\left(H_{l}\right.$ des Mann-Whitney-U-Tests ist anzunehmen bei $Z=-1.993, d_{\text {Cohens }}=.391, p=.046$ ), als auch des Lernerfolgs in der Dimension Erinnern $\left(H_{l}\right.$ bei $Z=-4.801$ anzunehmen, $d_{\text {Cohens }}=.87$, $p=.000$ ) signifikant über dem Lernerfolg der Lernenden in FunkO. Die Forschungshypothese $H_{1.1}$ kann für die Dimension Erinnern in beiden Studien so- 
wie über beide Studien aggregiert für den Lernerfolg insgesamt und das Erinnern angenommen werden; prozessorientiert Lernende erreichen hier einen höheren Lernerfolg.

Tab. 2: Lernerfolg in der funktions- und prozessorientierten Bedingung

\begin{tabular}{|c|c|c|c|c|c|c|}
\hline & \multirow{2}{*}{$\begin{array}{l}\text { Studie } 1 \\
\text { FunkO }\end{array}$} & \multicolumn{3}{|c|}{ Studie 2} & \multicolumn{2}{|c|}{ Studie 1 \& 2 aggr. } \\
\hline & & ProzO & FunkO & ProzO & FunkO & ProzO \\
\hline$n$ & 60 & 40 & 46 & 23 & 106 & 63 \\
\hline Lernerfolg $M$ & 10.10 & 11.20 & 11.14 & 14.87 & 10.55 & 12.54 \\
\hline$S D$ & 4.13 & 5.48 & 4.98 & 6.07 & 4.53 & 5.93 \\
\hline Kennwerte & \multicolumn{2}{|c|}{ n.s. } & \multicolumn{2}{|c|}{ n.s. } & \multicolumn{2}{|c|}{$\begin{array}{l}Z=-1.993, p=.046 \\
d=.391\end{array}$} \\
\hline \multirow{2}{*}{$\begin{array}{cc}\text { Erinnern } & M \\
& S \\
- & S\end{array}$} & 1.77 & 2.68 & 1.27 & 3.04 & 1.55 & 2.81 \\
\hline & 1.22 & 1.81 & 1.18 & 1.72 & 1.22 & 1.77 \\
\hline Kennwerte & \multicolumn{2}{|c|}{$\begin{aligned} Z & =-2.878, p=.004, \\
d & =.614\end{aligned}$} & \multicolumn{2}{|c|}{$\begin{array}{l}Z=-4.175, p=.000 \\
d=1.282\end{array}$} & \multicolumn{2}{|c|}{$\begin{array}{l}Z=-4.801, p=.000 \\
d=.87\end{array}$} \\
\hline Anwenden $M$ & 8.33 & 8.53 & 9.87 & 11.83 & 9.00 & 9.73 \\
\hline$S D$ & 3.82 & 4.58 & 4.60 & 5.88 & 4.22 & 5.30 \\
\hline Kennwerte & \multicolumn{2}{|c|}{ n.s. } & \multicolumn{2}{|r|}{ n.s. } & \multicolumn{2}{|c|}{ n.s. } \\
\hline
\end{tabular}

Quelle: eigene Darstellung

Zur zweiten Forschungsfrage: (2) Erreichen dyadisch Lernende einen höheren Lernerfolg als einzeln Lernende in Bezug auf das Erinnern und Anwenden des Gelernten? Für die Gruppen der dyadisch und einzeln Lernenden ist folgende Hypothese zu prüfen: $H_{2.1}$ Dyadisch Lernende erreichen einen höheren Lernerfolg als einzeln Lernende.

Für Studie 1 zeigen sich sowohl für den Lernerfolg insgesamt, als auch für die Dimensionen Erinnern und Anwenden bessere Lernergebnisse für dyadisch Lernende (Tab. 3). Diese Unterschiede sind signifikant mit kleinem bis mittlerem Effekt: für den Lernerfolg insgesamt $\left(H_{l}\right.$ des t-Tests ist bei $t(98)=-2.020$ anzunehmen, $\left.p=.046, d_{\text {Cohens }}=.404\right)$ sowie für die Dimension Erinnern $\left(H_{1}\right.$ des Mann-Whitney-U Tests ist bei $Z=-2.397$ anzunehmen, $d_{\text {Cohens }}=.465$, $p=.017)$. In Studie 2 erreichen die einzeln Lernenden insgesamt sowie im Anwenden einen höheren Lernerfolg. Diese Unterschiede zu den dyadisch Lernenden sind signifikant (Lernerfolg insgesamt: $H_{1}$ des t-Tests ist bei $t(67)=2.493$ anzunehmen, $p=.015, d_{\text {Cohens }}=.621$; Dimension Anwenden: $H_{1}$ des t-Tests ist bei $t(67)=3.010$ anzunehmen; $\left.p=.004, d_{\text {Cohens }}=.739\right)$. Für beide Studien aggregiert sind die deskriptiven Werte einzeln und dyadisch Lernender annähernd gleich (Tab. 3). Signifikant sind die Unterschiede zugunsten dyadisch Lernender für die Dimension Erinnern $\left(H_{l}\right.$ des Mann-Whitney-UTests ist bei $Z=-2.039$ anzunehmen, $\left.d_{\text {Cohens }}=.314, p=.041\right)$. Die Annahme 
der Forschungshypothese $H_{2 . I}$ kann für Studie 1 - für den Lernerfolg insgesamt und die Dimension Erinnern - sowie für die aggregierten Werte der Studien 1 und 2 für die Dimension Erinnern erfolgen.

Tab. 3: Lernerfolg der einzeln und dyadisch Lernenden

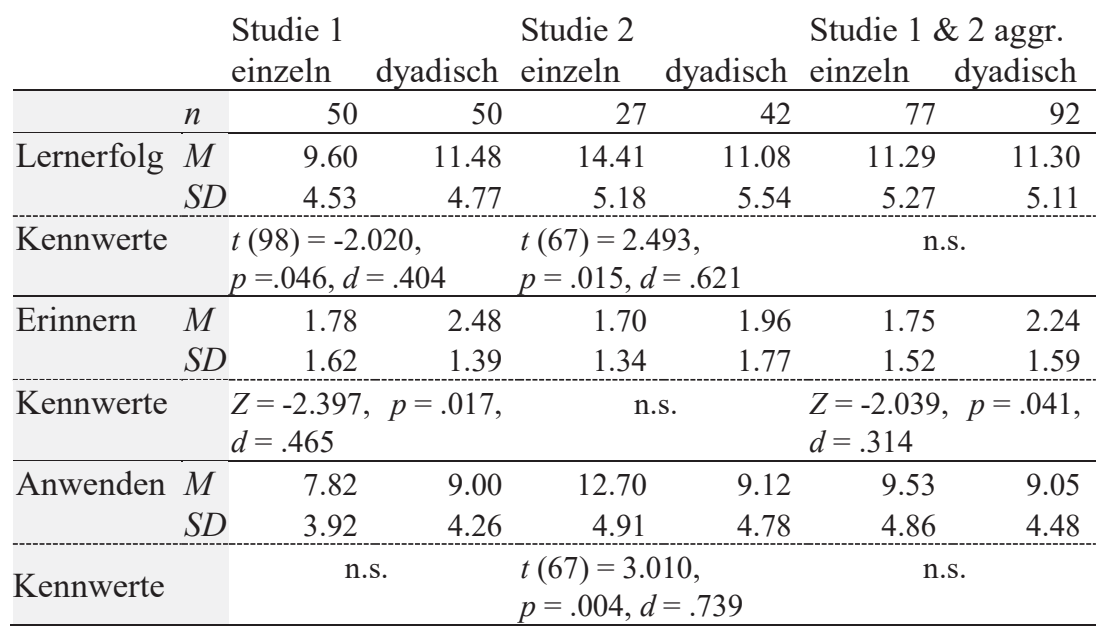

Quelle: eigene Darstellung

Zur dritten Forschungsfrage: (3) Erreichen einzeln Lernende in der Prozessorientierung einen höheren Lernerfolg als in der Funktionsorientierung? Für die Gruppen einzeln Lernender ist jeweils folgende Hypothese zu prüfen: $H_{3.1}$ Einzeln Lernende erreichen einen höheren Lernerfolg in der prozessorientierten als in der funktionsorientierten Bedingung.

Im Vergleich der einzeln Lernenden in den beiden Lernbedingungen zeigen sich in Studie 1 keine signifikanten Unterschiede, weder im Lernerfolg insgesamt, noch in den Dimensionen Erinnern oder Anwenden. Deskriptiv betrachtet, erreichen die einzeln Lernenden in ProzO in Studie 1 allerdings das insgesamt schlechteste Ergebnis $(M=9.02, S D=4.91)$ im Vergleich der vier Lernbedingungen (vgl. Tab. 4 und Tab. 5). Im Rahmen der Lehrveranstaltungen war zu beobachten, dass die einzeln Lernenden in ProzO beim Einstieg in die Problemsituation Schwierigkeiten im Umgang mit der Komplexität hatten. Die Orientierungsleistung, sich in der komplexen Problemstellung zurechtzufinden, schien hier - insbesondere im Vergleich zur dyadischen Bedingung in ProzO - besonders hoch. In Studie 2 wurden daher die einzeln Lernenden in ProzO in dieser Phase durch instruktionales Scaffolding (Belland 2017) unterstützt. Die Wirksamkeit des instruktionalen Scaffoldings zeigt sich auch im Lernerfolg der einzeln Lernenden in ProzO in Studie 2. Sie erreichen bessere 
Ergebnisse als in Studie 1 und schneiden auch im Vergleich zu einzeln Lernenden in FunkO besser ab (Tab. 4). Die vergleichsweise hohe Standardabweichung ist ein Indiz dafür, dass die einzeln Lernenden in ProzO unterschiedlich in der Lage sind, das Lernangebot effektiv zu nutzen. Die Vorteile zugunsten der einzeln Lernenden in ProzO gegenüber FunkO in Studie 2 zeigen sich als signifikante Unterschiede für die Dimension Erinnern mit großem Effekt $\left(H_{1}\right.$ des Mann-Whitney-U-Tests ist anzunehmen bei $Z=-3.057, d_{\text {Cohens }}=1.752$, $p=.001)$. Für die über Studie 1 und 2 aggregierten Werte zeigen sich signifikante Unterschiede in der Dimension Erinnern zugunsten einzeln Lernender in ProzO mit mittlerem Effekt ( $H_{l}$ des Mann-Whitney-U-Tests ist anzunehmen bei $Z=-2.198, d_{\text {Cohens }}=.585, p=.028$ ). Die Forschungshypothese $H_{3.1}$ kann nur für einzeln Lernende in der Dimension Erinnern in Studie 2 und für die aggregierten Werte angenommen werden.

Tab. 4: Lernerfolg der einzeln Lernenden

\begin{tabular}{|c|c|c|c|c|c|c|c|}
\hline & \multicolumn{2}{|l|}{ Studie 1} & \multicolumn{2}{|l|}{ Studie 2} & \multicolumn{2}{|c|}{ Studie $1 \& 2$ aggr. } \\
\hline & & FunkO & ProzO & FunkO & ProzO & FunkO & ProzO \\
\hline & $n$ & 29 & 21 & 19 & 8 & 48 & 29 \\
\hline \multirow[t]{2}{*}{ Lernerfolg } & $M$ & 10.02 & 9.02 & 13.11 & 17.50 & 11.24 & 11.36 \\
\hline & $S D$ & 4.27 & 4.91 & 3.57 & 7.17 & 4.25 & 6.70 \\
\hline \multicolumn{2}{|l|}{ Kennwerte } & \multicolumn{2}{|c|}{ n.s. } & \multicolumn{2}{|c|}{ n.s. } & \multicolumn{2}{|c|}{ n.s. } \\
\hline \multirow[t]{2}{*}{ Erinnern } & $M$ & 1.60 & 2.02 & 1.16 & 3.00 & 1.43 & 2.29 \\
\hline & $S D$ & 1.24 & 2.04 & .93 & 1.31 & 1.14 & 1.90 \\
\hline \multicolumn{2}{|l|}{ Kennwerte } & \multicolumn{2}{|c|}{ n.s. } & \multicolumn{2}{|c|}{$\begin{array}{l}Z=-3.057, p=.001, \\
d=1.752\end{array}$} & \multicolumn{2}{|c|}{$\begin{aligned} Z & =-2.198, p=.028, \\
d & =.585\end{aligned}$} \\
\hline \multirow[t]{2}{*}{ Anwenden } & $M$ & 8.41 & 7.00 & 11.95 & 14.50 & 9.81 & 9.07 \\
\hline & $S D$ & 3.95 & 3.82 & 3.63 & 7.09 & 4.17 & 5.89 \\
\hline \multicolumn{2}{|l|}{ Kennwerte } & \multicolumn{2}{|c|}{ n.s. } & \multicolumn{2}{|c|}{ n. } & \multicolumn{2}{|l|}{ 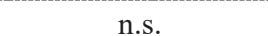 } \\
\hline
\end{tabular}

Quelle: eigene Darstellung

Zur vierten Forschungsfrage: (4) Erreichen dyadisch Lernende in der Prozessorientierung einen höheren Lernerfolg als in der Funktionsorientierung? Für die Gruppen dyadisch Lernender ist jeweils folgende Hypothese zu prüfen: $H_{4.1}$ Dyadisch Lernende erreichen einen höheren Lernerfolg in der prozessorientierten als in der funktionsorientierten Bedingung.

Dyadisch Lernende in ProzO erreichen sowohl in Studie 1, als auch in Studie 2 und über beide Studien aggregiert bessere Lernergebnisse als dyadisch Lernende in FunkO (Tab. 5). Für Studie 1 sind die Unterschiede zugunsten dyadisch Lernender in ProzO für den Lernerfolg insgesamt signifikant mit mittlerem Effekt $\left(H_{l}\right.$ des Mann-Whitney-U Tests ist anzunehmen bei $Z=-2.625$, 
$\left.p=.009, d_{\text {Cohens }}=.76\right)$; und in der Dimension Erinnern mit großem Effekt $\left(H_{I}\right.$ des Mann-Whitney-U Tests ist anzunehmen bei $Z=-3.670, p=.000$, $\left.d_{\text {Cohens }}=1.167\right)$. Für Studie 2 zeigen sich signifikante Unterschiede zugunsten dyadisch Lernender in ProzO in der Dimension Erinnern $\left(H_{l}\right.$ des Mann-Whitney-U Tests ist anzunehmen bei $\mathrm{Z}=-2.963, \mathrm{p}=.003, d_{\text {Cohens }}=1.091$ ). Für die aggregierten Daten beider Studien erreichen die Vorteile für dyadisch Lernende Signifikanzniveau sowohl hinsichtlich des Lernerfolgs insgesamt mit mittlerem Effekt, als auch in der Dimension Erinnern mit großem Effekt.

Tab. 5: Lernerfolg der dyadisch Lernenden

\begin{tabular}{|c|c|c|c|c|c|c|c|}
\hline & \multicolumn{2}{|l|}{ Studie 1} & \multicolumn{2}{|l|}{ Studie 2} & \multicolumn{2}{|c|}{ Studie $1 \& 2$ aggr. } \\
\hline & & FunkO & ProzO & FunkO & ProzO & FunkO & ProzO \\
\hline & $n$ & 31 & 19 & 27 & 15 & 58 & 34 \\
\hline \multirow{2}{*}{ Lernerfolg } & $M$ & 10.18 & 13.61 & 9.76 & 13.47 & 9.98 & 13.54 \\
\hline & $S D$ & 4.07 & 5.17 & 5.41 & 5.11 & 4.70 & 5.07 \\
\hline Kennwerte & \multicolumn{3}{|c|}{$\begin{array}{l}Z=-2.625, p=.009 \\
d=.76\end{array}$} & \multicolumn{2}{|c|}{$n$} & $\begin{array}{l}Z=-3.053 \\
d=.736\end{array}$ & $p=.002$, \\
\hline \multirow{2}{*}{ Erinnern } & $M$ & 1.92 & 3.39 & 1.35 & 3.07 & 1.66 & 3.25 \\
\hline & $S D$ & 1.20 & 1.19 & 1.34 & 1.94 & 1.29 & 1.55 \\
\hline Kennwerte & & \multicolumn{2}{|c|}{$\begin{array}{l}Z=-3.670, p=.000, \\
d=1.167\end{array}$} & \multicolumn{2}{|c|}{$\begin{array}{l}Z=-2.963, p=.003, \\
d=1.091\end{array}$} & \multicolumn{2}{|c|}{$\begin{aligned} Z & =-4.457, p=.000, \\
d & =1.143\end{aligned}$} \\
\hline \multirow{2}{*}{ Anwenden } & $M$ & 8.26 & 10.21 & 8.41 & 10.40 & 8.33 & 10.29 \\
\hline & $S D$ & 3.75 & 4.85 & 4.71 & 4.79 & 4.19 & 4.75 \\
\hline Kennwerte & & \multicolumn{2}{|c|}{ n.s } & \multicolumn{2}{|c|}{ n.s. } & \multicolumn{2}{|c|}{ n.s } \\
\hline
\end{tabular}

Quelle: eigene Darstellung

\section{Diskussion und Ausblick}

Dyadisch Lernende in geschäftsprozessorientiert gestalteten Lernprozessen zeigen durchgängig die besten Ergebnisse, was die Annahme einer förderlichen Wirkung handlungs- und problemorientierter Lernumgebungen unterstützt. Auffällig sind die in Studie 1 beobachteten Schwierigkeiten einzeln Lernender in der prozessorientierten Bedingung im Umgang mit der Komplexität der Lernsituation, die auf eine Überforderung durch die handlungs- und problemorientierte Lernumgebung hinweisen (vgl. Nickolaus 2007). Durch stärkere instruktionale Unterstützung in Form des Scaffoldings konnte diesen Schwierigkeiten in Studie 2 begegnet werden. Instruktionales Scaffolding ermöglicht individuelle und bedarfsgerechte Unterstützung (Belland 2017), um die Orientierung in der komplexen Lernsituation zu erleichtern. Die deutlich 
besseren Ergebnisse prozessorientiert einzeln Lernender in Studie 2 weisen auf die Wirksamkeit hin.

Insgesamt scheint die Prozessorientierung, die Situiertheit und Authentizität berücksichtigt, den Lernerfolg in der Dimension Erinnern stärker zu fördern als die Funktionsorientierung, sowohl bei einzeln als auch bei dyadisch Lernenden. Die Aussagen dyadisch Lernender in den retrospektiven Interviews, die Interaktion mit einem Lernpartner als hilfreich empfunden zu haben, bestätigen die angenommene positive Wirkung der Kooperation im Lernprozess. Die Beobachtung von King (2007) bestätigend, wurde in den retrospektiven Interviews ebenso deutlich, dass die Aufforderung zur Kooperation wichtig ist (,sonst hätte man sich weniger ausgestauscht', Proband FoD).

Limitierend für die Dimension Anwenden und deren Ergebnisse ist anzumerken, dass für einen sicheren Umgang mit ERP-Software mehr Zeit erforderlich ist, als die begrenzte Lernzeit im Rahmen der Erhebungen bieten konnte.

Prozessorientierte Unternehmen erwarten von ihren Mitarbeitenden ganzheitliches Prozessdenken. Den in praxi häufig anzutreffenden „Klickschulungen" ist zwar die Lernwirksamkeit nicht abzusprechen. Allerdings liefern die Aussagen der Probanden in den retrospektiven Interviews ergänzende Erkenntnisse, die u. a. die Annahme der fragmentierten Perspektive (Tramm 2009) bestätigen. Die ,starke Vorstrukturierung führt dazu, einfach nur abzuarbeiten “ (Proband FoD). Sechs Probanden (FunkO) empfanden die Klickanleitung als hinderlich für ihren Lernprozess. Probanden dieser Lernbedingung schlagen auch vor, das Vorgehen weniger stark vorzustrukturieren (,damit man sich mehr damit beschäftigt ${ }^{\prime}$ ) und wünschen sich ,eine komplexe Problemsituation, die aber mehr Zeit benötigen würde'.

Im Kontext des komplexen Forschungsprojekts sind weitere Erkenntnispotentiale zur Wirkung und Wirksamkeit der Geschäftsprozessorientierung als didaktischer Rahmen für Lernhandeln in SAP ERP HCM enthalten. Mithilfe der Item-Response-Theorie (IRT), speziell eines Partial Credit Modells (PCM), können den hier vorgestellten Daten zum Lernerfolg Item-Schwierigkeits- und Personenfähigkeitsparameter gegenübergestellt werden. Mögliche Zusammenhänge zu generalisierten Lernstrategien, Motivation sowie Erregung/Wohlbefinden während des Lernprozesses können aufgedeckt werden. Zusätzlich bieten die prozessbegleitend erhobenen Daten (wie die think-aloudProtokolle und Videodaten der Dyaden) Einblicke in das Lernverhalten und die Interaktionen während des Lernprozesses. Die Analyse dieser Daten erlaubt neben der vorgestellten lernerfolgsbezogenen Perspektive weitere differenzierte Erkenntnisse aus den kontrollierten Variablen, die zudem weitere Einblicke in das Lerngeschehen ermöglichen. 


\section{Literatur}

Achtenhagen, F., Tramm, T., Preiß, P., Seemann-Weymar, H., John, E. G. \& Schunck, A. (1992). Lernhandeln in komplexen Situationen. Neue Konzepte der betriebswirtschaftlichen Ausbildung. Wiesbaden: Gabler.

Anderson, L. W. \& Krathwohl, D. R. (2001). A Taxonomy for Learning, Teaching and Assessing. A Revision of Bloom's Taxonomy of Educational Objectives. New York: Longman.

Baheti, R. \& Gill, H. (2011). Cyber-physical systems. In T. Samad \& A. M. Annaswamy (Hrsg.), The impact of control technology. Overview, Success Stories, and Research Challanges (S. 161-166). Verfügbar unter http://ieeecss.org/main/IoCT-report [08.05.2018].

Belland, B. R. (2017). Instructional Scaffolding in STEM-Education. Strategies and Efficacy Evidence. Heidelberg, New York, Dordrecht, London: Springer.

Borsch, F. (2015). Kooperatives Lernen. Theorie - Anwendung - Wirksamkeit. Stuttgart: Kohlhammer.

Dehnbostel, P. (2007). Lernen im Prozess der Arbeit. Münster: Waxmann.

Dubs, R. (2009). Lehrerverhalten. Ein Beitrag zur Interaktion von Lehrenden und Lernenden im Unterricht. Stuttgart: Steiner.

Fischer, M. (2014). Arbeitsprozesswissen als Bezugspunkt für die Planung und Evaluation lernfeldorientierten Unterrichts. bwp@-Berufs- und Wirtschaftspädagogik - online. Verfügbar unter www.bwpat.de/profil3/ fischer profil3.pdf [18.09.2017].

Gaitanides, M. \& Ackermann, I. (2004). Die Geschäftsprozessperspektive als Schlüssel zu betriebswirtschaftlichem Denken und Handeln. bwp@ Spezial 1-2004. Verfügbar unter http://www.bwpat.de/spezial1/ gaitanides-acker.shtml [08.11.2017].

Gudjons, H. (2014). Handlungsorientiert lehren und lernen. Schüleraktivierung - Selbsttätigkeit-Projektarbeit (8. Aufl.). Bad Heilbrunn: Klinkhardt.

Hasselhorn, M. \& Gold, A. (2013). Pädagogische Psychologie. Erfolgreiches Lernen und Lehren (3. Aufl.). Stuttgart: Kohlhammer.

Hattie, J. (2012). Visible Learning for Teachers. Maximizing impact on learning. London, New York: Routledge.

Hilgenberg, B. (2014). Fehler bei der ERP-Einführung. Woran ERP-Projekte wirklich scheitern. Computerwoche. Verfügbar unter https://www.computerwoche.de/a/-woran-erpprojekte-wirklich-scheitern,2530844 [29.06.2017].

Hommel, M. (2017). Geschäftsprozess- und funktionsorientiertes Lernen am Beispiel von SAP ERP HCM. In K. Wilbers (Hrsg.), Industrie 4.0, Herausforderungen für die kaufmännische Bildung. Texte zur Wirtschaftspädagogik und Personalentwicklung, Band 19 (S. 155-185). Berlin: epubli.

Jansen, A., Müller, C., Prümper, J. \& Stein, B. (2005). Software-Einführung in KMU (kein) Platz für Benutzerbeteiligung - eine qualitative Bestandsaufnahme. In M. Hassenzahl \& M. Peissner (Hrsg.), Usability Professionals 2005 (S. 108-110). Stuttgart: German Chapter der Usability Professionals Association e.V.

King, A. (2007). Scripting Collaborative Learning Processes: A Cognitive Perspective. In F. Fischer, I. Kollar, H. Mandl \& J. M. Haake (Hrsg.), Scripting Computer-Supported Collaborative Learning: Cognitive, Computational and Educational Perspectives (S. 13-37). Boston: Springer. 
Klauser, F. (1998). Problem-based learning. Ein curricularer und didaktisch-methodischer Ansatz zur innovativen Gestaltung der kaufmännischen Ausbildung. Zeitschrift für Erziehungswissenschaft, 1(2), 273-293.

Konrad, K. (2014). Lernen lernen - allein und mit anderen. Konzepte, Lösungen, Beispiele. Wiesbaden: Springer.

Lou, Y., Abrami, P. C. \& d'Apollonia, S. (2001). Small Group and Individual Learning with Technology: A Meta-Analysis. Review of Educational Research, 71(3), 449-521.

Mayring, P. (2015). Qualitative Inhaltsanalyse. Grundlagen und Techniken (12. Aufl.). Weinheim, Basel: Beltz.

Nickolaus, R. (2007). Didaktik-Modelle und Konzepte beruflicher Bildung. Orientierungsleistungen für die Praxis. Baltmannsweiler: Schneider Verlag Hohengehren.

Pongratz, H. (2012). Implementierung von ERP-Systemen in den Unterricht an beruflichen Schulen (Dissertation). In K. Wilbers (Hrsg.), Texte zur Wirtschaftspädagogik und Personalentwicklung (Band 9). Nürnberg: epubli.

Rebmann, K. \& Schlömer, T. (2009). Lernen im Prozess der Arbeit. In A. Diettrich, D. Frommberger \& J. Klusmeyer (Hrsg.), bwp@ Profil 2, Berufs- und Wirtschaftspädagogik - online. Verfügbar unter http://www.bwpat.de/profil2/rebmann_schloemer_profil2.pdf [08.11.2017].

Rebmann, K. \& Tenfelde, W. (2008). Betriebliches Lernen. Mering: Hampp.

Renkl, A. \& Mandl, H. (1995). Kooperatives Lernen: Die Frage nach dem Notwendigen und dem Ersetzbaren. Unterrichtswissenschaft, 23(4), 292-300.

Reinmann, G. \& Mandl, H. (2006). Unterrichten und Lernumgebungen gestalten. In A. Krapp \& B. Weidenmann (Hrsg.) Pädagogische Psychologie (5. vollst. überarb. Aufl.), (S. 613-658). Weinheim: Beltz PVU.

Rost, D. (2013). Interpretation und Bewertung pädagogisch-psychologischer Studien (3., überarb. und erw. Aufl.). Bad Heilbrunn: Klinkhardt.

SAP (o. D.). IDES - das SAP Modellunternehmen. Verfügbar unter https://help-.sap.com/saphelp_46c/helpdata/de/af/fc4f35dfe82578e10000009b38f839/frameset-.htm [08.11.2017].

Sembill, D. \& Frötschl, C. (2018). Spannungsfelder digitalisierter Bildungswelten. In: J. Schlicht \& U. Moschner (Hrsg.), Berufliche Bildung an der Grenze zwischen Wirtschaft und Pädagogik (S. 159-178). Wiesbaden: Springer.

Tramm, T. (2009). Von der Geschäftsprozess- zur Lernprozessperspektive. In H. Pon-gratz, T. Tramm \& K. Wilbers (Hrsg.), Prozessorientierte Wirtschaftsdidaktik und Einsatz von ERP-Systemen im kaufmännischen Unterricht. Texte zur Wirtschaftspädagogik und Personalentwicklung, Band 4 (S. 77-101). Aachen: Shaker. 